\title{
FACTORS INFLUENCING IMPLEMENTATION OF EVIDENCE-BASED INTERVENTIONS IN PUBLIC HEALTH SYSTEMS - A MODEL
}

\author{
Joanne Vincenten ${ }^{1}$, J. Morag MacKay ${ }^{2}$, Peter Schröder-Bäck', Tamara Schloemer ${ }^{1}$, Helmut Brand ${ }^{1,3}$ \\ ${ }^{1}$ Department of International Health, School CAPHRI - Care and Public Health Research Institute, Faculty of Health, Medicine and Life \\ Sciences, Maastricht University, Maastricht, the Netherlands \\ ${ }^{2}$ Safe Kids Worldwide, Washington DC, United States of America \\ ${ }^{3}$ Prasanna School of Public Health, Manipal Academy of Higher Education, Manipal, India
}

\section{SUMMARY}

Objectives: Evidence that health interventions work is not enough to ensure implementation into policy and practice. The complexity of public health systems requires more diverse actions. This paper proposes a conceptual model to enhance understanding of interlinking factors that influence the evidence implementation process.

Methods: A literature review was conducted to explore factors that influence the process of evidence implementation in complex public health systems, including research findings and observations from 32 case studies of injury prevention interventions underway in 24 countries and results from evidence research networks. Concepts and themes identified through a critical review were organised, ordered and interlinked to build the model.

Results: An Evidence Implementation Model for Public Health Systems and four concepts or themes: Evidence implementation target; Actors involved in implementation; Knowledge transfer; and Barriers and facilitators to evidence implementation were developed to provide a simplified, yet broad framework that highlights multiple factors and back and forth inter-linkages within and between the concepts that influence the uptake of evidence into public health systems policy and practice.

Conclusions: Understanding the factors discussed within and amongst the four concepts of this model should ultimately help to positively influence the uptake of evidence into real world public health systems. This model has relevance for decision makers, researchers, knowledge brokers, and implementers.

Key words: evidence, implementation, decision-making, public health systems, knowledge transfer

Address for correspondence: J. Vincenten, Department of International Health, School CAPHRI - Care and Public Health Research Institute, Faculty of Health, Medicine and Life Sciences, Maastricht University, P.O. Box 616, 6200 MD Maastricht, the Netherlands. E-mail: joanne.vincenten@gmail.com

https://doi.org/10.21101/cejph.a5234

\section{INTRODUCTION}

Implementation of what has been demonstrated to work in public health should improve the health and wellbeing of society (1-3). However, the existence of evidence-based public health strategies itself does not seem to be convincing or compelling enough to have decision makers put evidence into practice (4-7). The complexity of public health systems requires more diverse actions (7-12).

In fact, several authors have reported barriers to achieving uptake of evidence within complex public health systems, including both policy and practice (12-14). Others have indicated the need for research beyond identifying barriers and have suggested examination of broader concepts, such as setting targets, actors involved in implementation, and knowledge transfer and translation that influence the processes of evidence implementation within public health systems as a whole $(2,8,9,14-17)$. The conceptual model described in this paper explores these broader factors and attempts to illustrate how they interlink and continually impact each other to influence the uptake of evidence into the development, implementation and monitoring of public health policies and practice $(10,18,19)$.

\section{MATERIALS AND METHODS}

A critical review was conducted. This type of review aims to identify the contribution of concepts to embody existing knowledge or derive a new theory. A core characteristic of a critical review is conceptual innovation (20). We used this methodology to explore the four concepts of Evidence implementation target; Actors involved in implementation; Knowledge transfer; and Barriers and facilitators that influence the process of evidence implementation in complex public health systems. This allowed us to understand, enrich and model these four concepts.

A key component of a critical review is to identify what is of value from the existing body of work (20). To identify recent relevant literature from both general and specific sources, we have 
searched PubMed and Google Scholar for articles published in English since 2010. The search terms used are listed in Box 1 and were used as single terms. As Grant and Booth (20) also address the value of specified sources, we also searched the websites of four institutions known for generating, preparing and translating the evidence base for policy making: the Evidence Informed Policy Network (EVIPNet) (21), the Health Evidence Network (HEN) (22), McMaster Health Forum-Health Systems Evidence (23), and the National Institute for Health and Care Excellence (NICE) (24). These institutional websites served to provide valuable literature (20) and specified knowledge for the topic. Articles were selected if they described elements of one or more of the four concepts and the reference lists of those articles were reviewed for additional relevant articles without regard to publication date. In addition to published articles, research findings of the European Commission funded project "Tools to Address Childhood Trauma, Injury and Children's Safety" (TACTICS) (25) were reviewed and relevant findings and the authors' project experiences were taken into consideration. This included 32 case studies of injury prevention interventions underway in 24 countries. We included the results of this multinational qualitative study as it provided practical information on the application of the four concepts of interest.

The selected articles and case studies were analysed with regard to the four concepts of interest. Relevant information, including connections between the four concepts was extracted, compared and ordered by concept. As recommended for the synthesis of extracted evidence in a critical review (20), we used these results to develop a model around the core concept of "evidence implementation." For this, key information describing themes of the concepts was organised, ordered and interlinked to build the model. The results and the subsequent model were discussed between all authors in order to best conceptualise what is relevant and of value for evidence implementation in complex public health systems.

Many of the terms encountered within the literature examining evidence implementation are used repeatedly, but with inconsistent definitions $(3,13,16)$. Therefore, brief working definitions were created by the authors to set a context for this work and are outlined below related to the circles of the model.

\section{RESULTS}

The Evidence Implementation Model for Public Health Systems (Fig. 1) was created to demonstrate the continuity and flow required for the implementation of evidence-based interventions in complex public health systems. The four concepts of the model: Evidence implementation target; Actors involved in implementation; Knowledge transfer; and Barriers and facilitators to evidence implementation are described below from inner to outer circle, including how they are interlinked.

\section{First Circle - Evidence Implementation Target}

To be successful at any given task, one needs to begin with the end in mind $(10,19)$. What is it that one wants to achieve? As a result, at the centre of the model is the desired target to be achieved through evidence implementation. For the purpose of this paper,

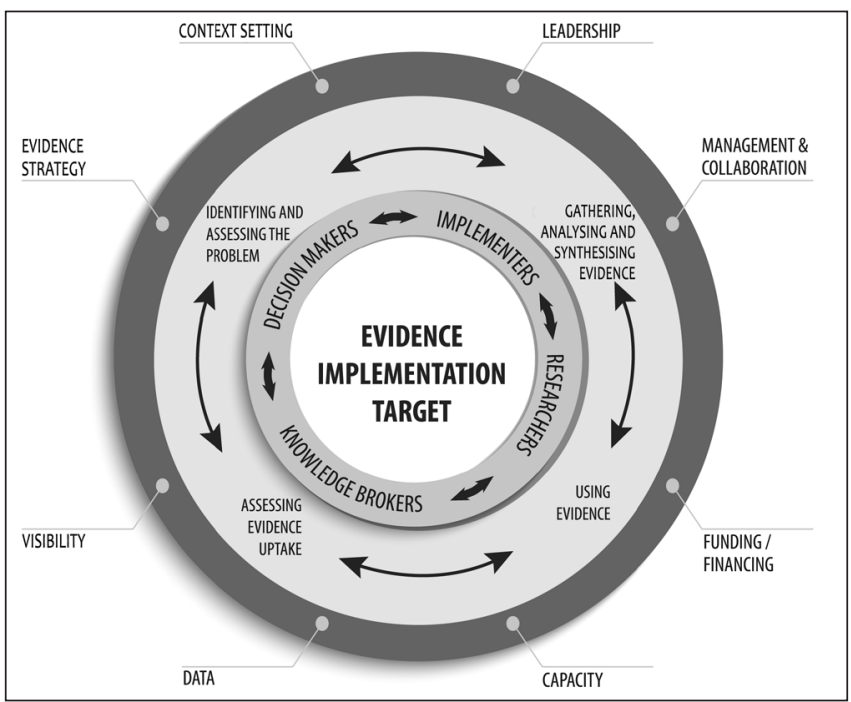

Fig. 1. Evidence implementation model for public health systems.

Circle 1 Evidence implementation target

Circle 2 Actors involved in implementation

Circle 3 Knowledge transfer

Circle 4 Barriers and facilitators to evidence implementation

evidence implementation was defined as the act of implementing or putting a set of specific actions in place to achieve a desired evidence-based intervention.

This target could be defined with either a qualitative and/or quantitative target measure, for the uptake of an evidenced-based intervention into policy or practice or both (e.g. introducing and enforcing minimum drinking age limits or forcing a reduction of speed around schools, residential areas, play areas).

\section{Second Circle - Actors Involved in Evidence Imple- mentation}

The second circle of the model reflects the key actors involved in the evidence implementation process $(17,26)$. Rather than proceeding as a linear process, a back and forth interplay between all actors continually occurs, ideally resulting in clarity, agreement and where necessary compromise. This indicates the need for on-going information exchange throughout the entire process, as well as emphasising the potential influence of both individual actors' professional and personal values, beliefs and preferences on the process, and the evidence implementation target $(3,14$, 15). The actors (described below) form the circle immediately adjacent to the evidence implementation target to highlight the importance of early, sustained and collaborative engagement of all key stakeholders in the evidence implementation process $(7$, 10, 13, 27).

Decision makers are those who decide on matters in public health systems and may be individuals, groups or organisations. Funders can often be part of the decision-making group directly, as may be the case for initiatives supported by governmental agencies (17). Funders may also have direct influence on those responsible for taking decisions, such as foundations that have set funding criteria $(15,19,26,28)$.

Researchers are those individuals or teams that systematically investigate a specific subject matter. The results of their research often serve as the basis for evidence-based interventions. Re- 
searchers may need to provide different kinds of evidence information for decision makers and implementers at various points in the implementation process $(29,30)$.

Knowledge brokers are a diverse set of individuals, groups or organisations that contribute to transitioning knowledge between researchers, decision makers and implementers, such as specialised applied research units, international governmental organisations, non-governmental organisations, media, think tanks, or consultancies. They help bridge the research to practice gap by sharing and translating knowledge, information, networks, tools, resources and capacity building means that contribute to the uptake of the evidence-based intervention $(1,13,14,31)$.

Implementers are those individuals and their respective organisations, as well as the public in general, that ultimately will be putting the evidence into practice in the real world. This includes addressing availability, accessibility and affordability of the intervention by the desired target audience (15).

Information moves back and forth between the actors, assisting them to gain further understanding, constantly adapt to change, solve problems, monitor results and consider further options and solutions to improve effectiveness and acceptability of delivery for the context, setting and target audience $(11,26)$.

\section{Third Circle - Knowledge Transfer}

The third circle of the model addresses knowledge transfer; that includes stages in a recursive process that has no set start or ending, but rather stages that are continually examined and addressed while moving back and forth between each of the stages: identifying and assessing the problem; gathering, analysing and synthesising evidence; using evidence; and assessing evidence uptake $(27,31-33)$.

Identifying and assessing the problem: to define a problem and determine the needed actions to achieve identified priorities.

Gathering, analysing and synthesising evidence: to systematically undertake investigation to attain best available and preferably local information to support decision-making processes and then to combine and summarise gathered evidence into an accessible and condensed format.

Using evidence: to translate, disseminate and apply evidence. Translate evidence: transfer and adapt the key facts and concepts of the synthesised research evidence and integrate these strategically into targeted tools, resources, and processes for each of the identified target audiences. Disseminate evidence: to distribute evidence and its translated versions in the form of tools, resources, and processes that maximise the target audiences' exposure to the results, key messages, outputs and outcomes, and supports their uptake. Apply evidence: the stage of taking up the stated evidence, adapting, integrating and sustaining its basis into the real world setting with the support of the target audience appropriate tools, resources and processes.

Assessing evidence uptake: the interpretation and evaluation of the level of uptake of the evidence-based intervention into policy or practice to determine a range of lessons learned and benefits that have or have not been achieved (e.g., increased knowledge, positive behaviour change, health improvement, cost reductions, sustainability).

The knowledge transfer process is adjacent to the actor's circle in this model to highlight the strong relationships of the different actors to various stages of this process. Not only is it important to involve all the stakeholders early in the knowledge transfer process to enhance the opportunity for evidence implementation, but maintenance of that involvement throughout the process increases the likelihood of success $(1,28)$. For example, continued engagement of researchers to support the accurate translation of research evidence during the formation of targeted tools and their communication, dissemination and application is an important action beyond peer reviewed publication $(29,31)$. Active engagement of all actors in all stages of knowledge transfer should broaden the knowledge, understanding, values and perspectives of the key actors regarding the evidence-based intervention; support the management of barriers in the process that could derail the intended policy or practice; and guide collective work to enhance facilitators to support the uptake of the evidence-based intervention, described in the next circle of the model (10). Double headed arrows are used between the stages to represent the constant interchange, revisiting and linking that occur throughout knowledge transfer.

\section{Fourth Circle - Barriers and Facilitators to Evidence Implementation}

The outermost circle of the model is a diverse set of factors that at any point in the knowledge transfer process can influence the success of evidence implementation. These include leadership, management and collaboration, funding, capacity, data, visibility of the issue being addressed, the evidence-based intervention itself and the context setting. Each of these eight factors, identified through a systematic analysis of 32 case studies from 24 countries examining evidence uptake, implementation and monitoring of interventions to prevent child injury, can present potential barriers to the process, yet if effectively managed, can facilitate success (25). For example, the actions of a competent leader can bring together diverse stakeholders towards a common vision and targets, while the absence of leadership can leave policies and practice with no direction or momentum $(29,31)$. Sound management and collaboration, including ensuring the right partners with clearly delineated roles and responsibilities, will support implementation (34). On the other hand, a lack of communication and or collaboration can bring implementation to a grinding halt (13). Without adequate funding to implement an intervention, the challenges to successfully reach the implementation target become daunting (34). Lack of funding creates a never-ending treadmill to find financial resources, which consumes time and human resources needed for technical aspects of policy and practice work (30). Having sufficient work force with the right capacities is also required to effectively move through the knowledge transfer process (35). Capacity deficiencies create gaps in needed skill sets and trigger further barriers (2). Data are also essential for planning, implementing and monitoring (30). Without adequate, accurate data, there will be little opportunity to demonstrate the effectiveness of the evidence being implemented and make the case for further investment. In addition, some level of visibility of the issue and the evidence-based intervention selected as the "best solution" is required to raise further awareness, garner support and stimulate a call to action $(1,30,36)$.

It is also important to recognise that the evidence-based intervention itself can be a facilitator or barrier (18). It is essential that the fit between the intervention and context and setting into 
which it will be introduced has been examined, re-examined and found to be appropriate, i.e. the intervention is acceptable and adaptable to the context and setting (33).

\section{DISCUSSION}

\section{What Is Already Known on This Topic?}

Improving public health outcomes through successful uptake of evidence-based interventions is not a simple or quick task (1, $2,9,10,15)$. This is in large part because public health systems are complex $(4,5,8,12)$. There are many barriers and interdependencies involved that are embedded in the system as a whole, as noted in reviews of implementation frameworks and models $(16,32)$. Addressing the complexity of such systems requires an understanding of the system dynamics including the many actors, factors and stages influencing the entire system $(4,11,15)$.

\section{What Does This Study Add?}

This model provides a broad, integrated and practical approach to begin to understand the complexity of such systems as they relate to evidence implementation by examining the interrelationship of four conceptual circles representing key aspects of implementation identified in the scientific literature. While initially developed using case studies from child injury, the model has since been used by the lead author within World Health Organization projects $(37,38)$. This model has differences over other existing implementation frameworks and knowledge transfer models by not only demonstrating the theoretical complexities of knowledge translation (9), but also provides some practical guidance. This includes more focus on a practical approach to operationalise and work with the interplay between the factors influencing implementation, such as setting agreed upon targets, early and sustained engagement of diverse actors and addressing context specific barriers and facilitators identified within this model (5, $8,10,16,27,32,33)$.

Regarding the role of actors in evidence implementation, Greenhalgh et al.'s model (17) and Damschroder et al.'s framework (13) note the importance that individuals, in addition to organisations, can play in implementation processes. However, they do not directly include individual actors in their models

\section{Box 1: Search terms}

Search words were used as single terms and included the following:

evidence-based, facilitators, barriers, implementation, knowledge transfer, knowledge translation, implementation strategies, evidence implementation, research communication, public understanding of science, evidence research impact, communications theory, implementation models, policy models, complex adaptive systems, evidence implementation in public health systems, policy theory, systems thinking, complexity theory, critical theory, theory of change, complex adaptive systems theory, public health policy, policy-making and health policy. or acknowledge the key role that their experience and biases can contribute to the public health system as a whole. Several frameworks and models authors have noted the importance of researchers and decision makers working together to enhance the uptake of evidence $(14,39)$. However, it must be emphasised that within the field of public health, an even broader set of actors need to be regularly engaged to increase the likelihood of success $(6,8,25,37,38)$. Therefore, this model also includes knowledge brokers and implementers, whose role is to fill information gaps/facilitate communication between researchers and policy makers, support informed decisions and implement practical actions $(28,33)$.

The eight key barriers/facilitators included in the model were both identified through an analysis of 32 case studies of active interventions conducted by some of the authors (25), as well as verified in the literature $(14,17,30,35)$. The breadth of barriers/ facilitators, that can influence the uptake of evidence at any point during knowledge transfer, have not been specifically identified within a number of other implementation and knowledge transfer frameworks and models $(9,10,16,19,27,32,33)$. The barriers/ facilitators inclusion is to encourage the proactive identification and resolution of barriers and maximisation of facilitators throughout the process as practical guidance and essential actions in real world settings $(9,25,37,38)$. The barriers/facilitators are not necessarily independent of one another - i.e. there is interplay and co-dependencies also between them $(37,38)$. For example, funding and resources will relate to and be dependent on the intervention itself, as well as the context and setting into which it is to be implemented.

Actors' values, beliefs and preferences can directly influence evidence implementation, highlighting the critical need to purposively target tools and resources, their dissemination and application to the appropriate audiences at key times within the implementation process $(5,8,37,38)$. This is particularly true, where for example decision makers need and prefer brief, plain and common language policy support tools that include specific evidence for the relevant stage of the policy cycle (e.g., risk and impact assessments or start up, versus sustainability costs) $(1,14$, $25,28-31)$. While policy support tools, such as policy briefs may be based on systematic reviews or other peer reviewed publications, putting them in a concise, engaging and relevant format, with competent facts and values, specific to the target audience, greatly increases their utility $(36,39)$.

\section{Limitations of This Study}

Some limitations must be kept in mind when viewing this model. Presently the Evidence Implementation Model for Public Health Systems is at the conceptual stage. First, the authors compiled many of the definitions used within the four concepts, as one definitive resource defining all relevant concepts has not been acknowledged at this time. Further, while the model is based in part on an analysis of 32 case studies of interventions in 24 countries and builds on empirical findings through a critical review of the literature, the model has had limited application. The model has found its first applications and use $(37,38)$, yet requires further testing and validation in practice. Finally, the critical review was limited to English language documents and thus some bias may have been introduced. 


\section{CONCLUSIONS}

Evidence-based public health interventions exist, but in many cases their uptake, implementation and monitoring need to be enhanced in order to achieve greater health benefits for all. This Evidence Implementation Model for Public Health Systems provides a broad, integrated and practical framework to help decision makers, researchers, knowledge brokers and implementers identify opportunities to strengthen needed action. This model builds on previous models by identifying additional concepts that need consideration and providing more specific practical guidance through the identification of the key factors where action is needed to enhance implementation. A question the model raises is how do we attain a better understanding of the influence of key actors' values, beliefs and preferences on the various stages of the process in order to successfully support their needs and thereby increase evidence implementation and improve public health systems for both policy and practice. The model has implications for research, policy and practice that emphasises: the importance of setting measurable evidence implementation targets to focus action; the need for early, sustained collaboration of researchers, decision makers, knowledge brokers and implementers; the importance to engage all actors in all stages of knowledge transfer to increase shared aim and reduce barriers; that context specific barriers can continually effect evidence implementation, yet if adequately addressed can become facilitators; and that a broad, yet simplified framework supports real world evidence implementation.

\section{Conflict of Interests}

None declared

\section{REFERENCES}

1. Liverani M, Hawkins B, Parkhurst JO. Political and institutional influences on the use of evidence in public health policy. A systematic review. PLoS One. 2013;8(10):e77404. doi: 10.1371/journal.pone.0077404.

2. Orton L, Lloyd-Williams F, Taylor-Robinson D, O'Flaherty M, Capewell $\mathrm{S}$. The use of research evidence in public health decision making processes: systematic review. PLoS One. 2011;6(7):e21704. doi: 10.1371/ journal.pone.0021704.

3. Oliver K, Lorenc T, Innvær S. New directions in evidence-based policy research: a critical analysis of the literature. Health Res Policy Syst. 2014;12:34. doi: 10.1186/1478-4505-12-34.

4. Holmes BJ, Best A, Davies H, Hunter D, Kelly MP, Marshall M, et al. Mobilising knowledge in complex health systems: a call to action. Evidence Policy. 2017;13(3):539-60.

5. Rycroft J. From linear to complicated to complex, comment on "Using complexity and network concepts to inform healthcare knowledge translation". Int J Health Policy Manag. 2018 Jun;7(6):566-8.

6. Straus SE, Tetroe JM, Graham ID. Knowledge translation is the use of knowledge in health care decision making. J Clin Epidemiol. 2011 Jan;64(1):6-10.

7. Hanson D, Allegrante JP, Sleet DA, Finch CF. Research alone is not sufficient to prevent sport injuries. Br J Sports Med. 2014;48(8):682-4.

8. Kitson A, Brook A, Harvey G, Jordan Z, Marshall R, O'Shea R, et al Using complexity and network concepts to inform healthcare knowledge translation. Int J Health Policy Manag. 2018;7(3):231-43.

9. Nilsen P. Making sense of implementation theories, models and frameworks. Implement Sci. 2015;10:53. doi: 10.1186/s13012-015-0242-0.

10. Glasgow RE, Green LW, Taylor MV, Stange KC. An evidence integration triangle for aligning science with policy and practice. Am J Prev Med. 2012;42(6):646-54.

11. Best A, Holmes B. Systems thinking, knowledge and action: towards better models and methods. Evidence Policy. 2010;6(2):145-59.
12. Cairney P. Complexity theory in political science and public policy. Polit Stud Rev. 2012;10:346-58.

13. Damschroder LJ, Aron DC, Keith RE, Kirsh SR, Alexander JA, Lowery JC. Fostering implementation of health services research findings into practice: a consolidated framework for advancing implementation science. Implement Sci. 2009;4:50. doi: 10.1186/1748-5908-4-50.

14. Oliver K, Innvar S, Lorenc T, Woodman J, Thomas J. A systematic review of barriers to and facilitators of the use of evidence by policymakers. BMC Health Serv Res. 2014;14:2. doi: 10.1186/1472-6963-14-2.

15. Head BW. Reconsidering evidence-based policy: key issues and challenges. Policy Soc. 2010;29(2):77-94.

16. Moulin J, Sabater-Hernández D, Fernandez-Llimos F, Benrimoj S. A systematic review of implementation frameworks of innovations in healthcare and resulting generic implementation framework. Health Res Policy Syst. 2015;13:16. doi: 10.1186/s12961-015-0005-z.

17. Greenhalgh T, Robert G, Macfarlane F, Bate P. Kyriakidou O. Diffusion of innovations in service organizations: systematic review and recommendations. Milbank Q. 2004;82(4):581-629.

18. Kreindler SA. What if implementation is not the problem? Exploring the missing links between knowledge and action. Int J Health Plann Manage. 2016 Apr;31(2):208-26.

19. Aarons GA, Hurlburt M, Horwitz SM. Advancing a conceptual model of evidence-based practice implementation in public service sectors. Adm Policy Ment Health. 2011;38(1):4-23.

20. Grant MJ, Booth A. A typology of reviews: an analysis of 14 review types and associated methodologies. Health Info Libr J. 2009;26(2):91-108.

21. World Health Organization. Evidence Informed Policy Network [Internet]. Geneva: WHO [cited 2019 Jul 15]. Available from: http://www. euro.who.int/en/data-andevidence/evidence-informed-policy-making/ evidence-informed-policy-network-evipnet.

22. World Health Organization. Health Evidence Network (HEN) [Internet]. Geneva: WHO [cited 2019 Jul 15]. Available from: http:/www.euro. who.int/en/data-and-evidence/evidenceinformed-policy-making/healthevidence-network-hen.

23. Health Systems Evidence. About HSE [Internet]. HSE [cited 2019 Jul 15]. Available from: https://www.healthsystemsevidence.org/about?lang=en.

24. National Institute for Health and Care Excellence [Internet]. NICE [cited 2019 Jul 15]. Available from: https://www.nice.org.uk.

25. Scholtes B, Schröder-Bäck P, MacKay JM, Vincenten J, Förster K, Brand $\mathrm{H}$. Facilitators and barriers for the adoption, implementation and monitoring of child safety interventions: a multinational qualitative analysis. Inj Prev. 2017 Jun;23(3):197-204.

26. Smits PA, Denis JL. How research funding agencies support science integration into policy and practice: an international overview. Implement Sci. 2014;9:28. doi: 10.1186/1748-5908-9-28.

27. Grimshaw JM, Eccles MP, Lavis JN, Hill SJ, Squires JE. Knowledge translation of research findings. Implement Sci. 2012;7:50. doi: 10.1186/17485908-7-50.

28. Milat AJ, King L, Newson R, Wolfenden L, Rissel C, Bauman A, et al. Increasing the scale and adoption of population health interventions: experiences and perspectives of policy makers, practitioners, and researchers. Health Res Policy Syst. 2014;12:18. doi: 10.1186/1478-4505-12-18.

29. Innis J, Dryden-Palmer K, Perreira T, Berta W. How do health care organizations take on best practices? A scoping literature review. Int J Evid Based Health. 2015;13(4):254-72.

30. Humphries S, Stafinski T, Mumtaz Z, Menon D. Barriers and facilitators to evidence-use in program management: a systematic review of the literature. BMC Health Serv Res. 2014;14:171. doi: 10.1186/1472-696314-171.

31. Yost J, Dobbins M, Traynor R, DeCorby K, Workentine S, Greco L. Tools to support evidence-informed public health decision making. BMC Public Health. 2014;14(1):728-41. doi: 10.1186/1471-2458-14-728.

32. Field B, Booth A, Ilott I, Gerrish K. Using the knowledge to action framework in practice: a citation analysis and systematic review. Implement Sci. 2014;9:172. doi 10.1186/s13012-014-0172-2.

33. Ingold $\mathrm{J}$, Monaghan M. Evidence translation: an exploration of policy makers' use of evidence. Policy Politics. 2016;44(2):171-90.

34. Gagliardi AR, Webster F, Brouwers MC, Baxter NN, Finelli A, Gallinger $\mathrm{S}$. How does context influence collaborative decision-making for health services planning, delivery and evaluation? BMC Health Serv Res. 2014; 14:545. doi: 10.1186/s12913-014-0545-x.

35. Ellen ME, Léon G, Bouchard G, Ouimet M, Grimshaw JM, Lavis JN. Barriers, facilitators and views about next steps to implementing supports for evidence informed decision-making in health systems: a qualitative study. Implement Sci. 2014;9:179. doi: 10.1186/s13012-014-0179-8. 
36. Dietz T. Bringing values and deliberation to science communication. Proc Natl Acad Sci U S A. 2013 Aug 20;110 Suppl 3:14081-7.

37. Vincenten J, George F, Martuzzi M, Schröder-Bäck P, Paunovic E. Barriers and facilitators to the elimination of asbestos related diseasesstakeholders' perspectives. Int J Environ Res Public Health. 2017 Oct 22;14(10). doi: 10.3390/ijerph14101269.

38. World Health Organization. Analysis of stakeholder views on future development in chemical safety in the WHO European Region [Internet]. Copenhagen: WHO Regional Office for Europe; 2017 [cited 2019 Jul 15]. Available from: http://www.euro.who.int/ data/assets/ pdf_file/0016/340270/Stakeholder-Analisys_WHO_web3.pdf.
39. Lavis J, Moynihan R, Oxman A, Paulsen E. Evidence-informed health policy 4 - Case descriptions of organizations that support the use of research evidence. Implement Sci. 2008;3:56. doi: 10.1186/1748-5908$3-56$.

Received October 17, 2017 Accepted in revised form July 23, 2019 